\title{
Sistemas DS/CDMA Multitaxa com Detector Heurístico-Genético em Canais Multipercurso
}

\section{Multirate DS/CDMA Systems with Heuristic-Genetic Detector in Multipath Channels}

\author{
Fernando Ciriaco $^{1}$; Taufik Abrão ${ }^{2}$; Paul Jean E. Jeszensky ${ }^{3}$
}

\section{Resumo}

Este trabalho analisa uma técnica de otimização combinatória baseada em algoritmo genético (GA) aplicada ao problema da detecção multiusuário (MuD) em sistemas DS/CDMA multitaxa por códigos múltiplos (MC) em canais com desvanecimento multipercurso. Resultados de desempenho via simulação Monte Carlo (MCS), em duas situações de carregamento multitaxa, indicaram convergência próxima ao limite do detector multiusuário ótimo $(\mathrm{OMuD})$. A complexidade computacional do algoritmo genético, em termos do número de operações necessárias para se atingir a convergência, é encontrada e comparada à do OMuD, indicando um expressivo ganho em termos de compromisso complexidade-desempenho em relação ao receptor convencional (Rake).

Palavras-chave: Algoritmo Genético. Códigos Múltiplos. Detecção Multiusuário. DS/CDMA. Esquemas Multitaxa. Detector Ótimo.

\begin{abstract}
This work analyses a combinatorial optimization technique based on genetic algorithm (GA) applied to the multiuser detection problem (MuD) in multirate DS/CDMA systems by multiple codes in multipath fading channels. Monte Carlo simulation (MCS) results, in two multirate conditions, showed that the detection based on GA is a viable option when compared with the optimum MuD (OMuD). The genetic algorithm complexity is determined and compared with the $\mathrm{OMuD}$ based on the required number of computational operations, showing an expressive improvement in terms of complexity-performance tradeoff when compared with Rake receiver.

Key words: Genetic Algorithm. DS/CDMA. Multiple Codes. Multirate. Multiuser Detection. Optimum Detector.
\end{abstract}

\footnotetext{
${ }^{1}$ Aluno de mestrado do Departamento de Engenharia Elétrica, Universidade Estadual de Londrina (DEEL-UEL); fernandociriaco@ sercomtel.com.br

2 Professor Adjunto do DEEL-UEL; taufik@uel.br

${ }^{3}$ Professor Titular da Escola Politécnica da USP, PTC; pjj@1cs.poli.usp.br
} 


\section{Introdução}

Nos últimos anos os serviços de comunicação móvel vêm sofrendo grandes mudanças para poder atender a explosão na demanda de serviços de áudio, dados e vídeo. Para que esses serviços sejam oferecidos com qualidade, grupos de pesquisas em todo o mundo vêm fazendo grandes esforços na busca de sistemas de comunicação sem fio de alta eficiência espectral com capacidade e desempenhos melhorados, quer seja em termos do número máximo de usuários por unidade de área suportado em uma mesma banda, quer seja na obtenção de sistemas móveis capazes de atender essa diversidade de serviços. A terceira geração de sistemas móveis celulares (3G) visa dar suporte a tais serviços, através do uso de esquemas de transmissões com taxas variadas e distintas exigências de qualidade de serviço (QoS). Com isso, os sistemas 3G devem acomodar usuários transmitindo simultaneamente a distintas taxas em canais com tráfego assimétrico (i.e., enlaces direto e reverso podem ser requisitados a trabalharem em taxas distintas), e ainda garantir as especificações mínimas de QoS para cada um dos serviços oferecidos. Esquemas de transmissão (ou de acesso) de múltipla taxa (ou multitaxa) buscam viabilizar o tráfego de serviços multimídia com menor complexidade e máxima eficiência possíveis. Dentre os quatro esquemas de transmissão multitaxa básicos (ABRÃO, 2001) destaca-se o de códigos múltiplos (MC) por sua simplicidade de implementação. Na estratégia de acesso de múltipla taxa por código múltiplo, também denominada canais paralelos, todos os usuários multiplexam seus bits de informação utilizando várias sequiências de espalhamento, transmitindo seus bits de informação paralela e sincronamente (JOHANSSON, 1998). Assim, todos os canais paralelos de um determinado usuário estarão sujeitos às mesmas condições de canal, i.e. às mesmas condições de desvanecimento e de atrasos de propagação. Com isso, todos os usuários terão o mesmo ganho de processamento, possibilitando o uso de um conjunto de sequiências de espalhamento com boas propriedades de correlação cruzada. Para isso, emprega-se o conjunto de seqüências de espalhamento ortogonais, como as de Walsh-Hadamard e OVSF em sistemas 3G, amenizando o efeito da elevada interferência de múltiplo acesso.

Este trabalho analisa a aplicação do algoritmo genético na detecção multiusuário de sistemas multitaxa por códigos múltiplos (GA-MC-MuD) em canais com desvanecimento multipercurso. $\mathrm{O}$ método de otimização combinatória baseado no princípio da evolução genética, aplicado ao problema de detecção multiusuário, visa atingir o desempenho ótimo ou quase-ótimo com reduzida complexidade computacional, ou seja, factível de implementação quando comparado ao $\mathrm{OMuD}$, resultando assim em um expressivo aumento de capacidade do sistema em relação à detecção convencional (receptor Rake), com garantia de QoS dos diferentes serviços multitaxa.

A seguir, descreve-se o modelo do sistema DS/CDMA multitaxa por códigos múltiplos em canais com desvanecimento multipercurso e também o problema $\mathrm{MuD}$ a ser otimizado. Na seção seguinte, faz-se uma descrição genérica do GA-MC$\mathrm{MuD}$, apresentando as estratégias adotadas para as diferentes etapas do algoritmo genético. Os resultados numéricos de desempenho para o GA-MC-MuD, em termos de taxa de erro de bit (BER), são mostrados na seção Resultados Numéricos. A complexidade computacional do OMuD e do GA-MC-MuD, em termos do número de operações computadas na otimização da função custo, é discutida em seguida. Finalmente, são sintetizadas as principais conclusões deste trabalho. 


\section{Modelo de Sistema DS/CDMA}

A figura 1 esquematiza o transmissor e receptor para um sistema MC DS/CDMA em canal com desvanecimento multipercurso e detecção utilizando o algoritmo GA-MC-MuD proposto. Os bits transmitidos pelo $k$-ésimo usuário pertencente ao $g$-ésimo grupo multitaxa MC, de um total de $G$ grupos, utilizandose de $m^{(g)}=\frac{R^{(g)}}{R}$ códigos paralelos no intervalo de bit $T$, são:

$$
\mathbf{b}_{k}^{(g)}=\left[b_{k, 1}, b_{k, 2}, \ldots b_{k, m^{(g)}}\right]^{\top}
$$

com $R^{(g)}=$ taxa de dados do $g$-ésimo grupo multitaxa e $R=\frac{1}{T}=$ taxa básica, admitida sub-múltipla inteira de $R^{(g)}$.

Os símbolos de informação são espalhados em duas etapas. A canalização permite que dados de canais paralelos de um mesmo usuário sejam ortogonais; já a etapa de embaralhamento permite obter uma certa rejeição de interferência de múltiplo acesso, além de permitir a identificação de cada usuário DS/CDMA. Assumindo-se que o $k$-ésimo usuário MC emprega modulação BPSK sobre as $m^{(g)}$ formas de ondas, o sinal transmitido em banda base após o espalhamento de canalização pode ser expresso por (ABRÃO, 2001):

$$
u_{k}^{(g)}(t)=\sum_{i}^{m^{(g)}} \mathbf{b}_{k}^{(g)}(i) \tilde{\mathbf{s}}_{C k}^{(g)}(t-i T)
$$

onde o vetor forma de onda do código de canalização normalizado para o $k$-ésimo usuário é definido por:

$\tilde{\mathbf{s}}_{C k}^{(g)}(t)=\frac{1}{\sqrt{N_{C}}}\left[s_{C k, 1}^{(g)}(t), s_{C k, 2}^{(g)}(t), \ldots, s_{C k, m^{(g)}}^{(g)}(t)\right]$

sendo o $m$-ésimo código de canalização do $k$-ésimo usuário, de comprimento $N_{C}$, dado por:

$$
s_{C k, m}^{(g)}(t)=\sum_{i=0}^{N_{C}-1} \underline{s}_{C, m}^{(g)} p_{T_{c h}}\left(t-i T_{c h}\right)
$$

onde $\underline{s}_{C}^{(g)}(\mathrm{m}) \in\{ \pm 1\}$ são os elementos do vetor de chips de canalização formatado, $T_{c h}$ é o intervalo de chip de canalização e $p_{T_{c h}}($.$) é a formatação de$ pulso de canalização, definido no intervalo $\left[0, T_{c h}\right)$. Admite-se aqui que para todos os usuários $\mathrm{MC}$, o respectivo vetor de chips de canalização (4) é designado a partir de um único conjunto de seqüências de espalhamento Walsh-Hadamard (W-H), de comprimento $N_{C} \geq m^{(G)}$, atribuindo-se os primeiros $m^{(g)}$ códigos ao $k$-ésimo usuário de taxa $m^{(g)} R$. Uma vez que para o mesmo usuário $\mathrm{MC}$ a condição de fase preferencial entre as seqüências do W-H é mantida, a interferência gerada será virtualmente zero. A seguir, realiza-se a etapa de embaralhamento utilizando-se de seqüências de espalhamento pseudo-aleatórias (PN); para o $k$-ésimo usuário multitaxa, o sinal em banda passante após o segundo espalhamento resulta:

$$
x_{k}^{(g)}(t)=\sqrt{2 P_{k}} \sum_{j} u_{k}^{(g)} s_{k}(t-j T) \cos \left(\omega_{c} t\right)
$$

onde $P_{k}=A_{k}^{2} / 2$ representa a potência de transmissão do $k$-ésimo usuário multitaxa; $\omega_{c}$ é a frequência da portadora; $s_{k}(t)$ corresponde à sequência de embaralhamento do $k$-ésimo usuário definida no intervalo $[0, T)$ e zero fora:

$$
s_{k}(t)=\frac{1}{\sqrt{N_{P N}}} \sum_{n=0}^{N_{P N}-1} p_{T_{c}}\left(t-n T_{c}\right) \underline{s}_{k, n}
$$

onde $\underline{s}_{k, n} \in\{ \pm 1\}$ é o $n$-ésimo chip da seqüência PN de comprimento $N_{P N}=\frac{T}{T_{c}}$ utilizada pelo $k$ ésimo usuário; $T_{c}$ é o período de chip que define a largura de banda do sistema DS/CDMA; ambas as formatações de pulso, $p_{T_{c}}$ e $p_{T_{c h}}$, são assumidas retangulares com amplitude unitária no intervalo $\left[0 ; T_{c}\right)$ e $\left[0 ; T_{c h}\right)$ e zero fora, respectivamente. Considerando o enlace reverso e assumindo um conjunto de bits transmitidos (frame) consistindo de $I$ bits para cada usuário MC propagando-se através de $L$ 


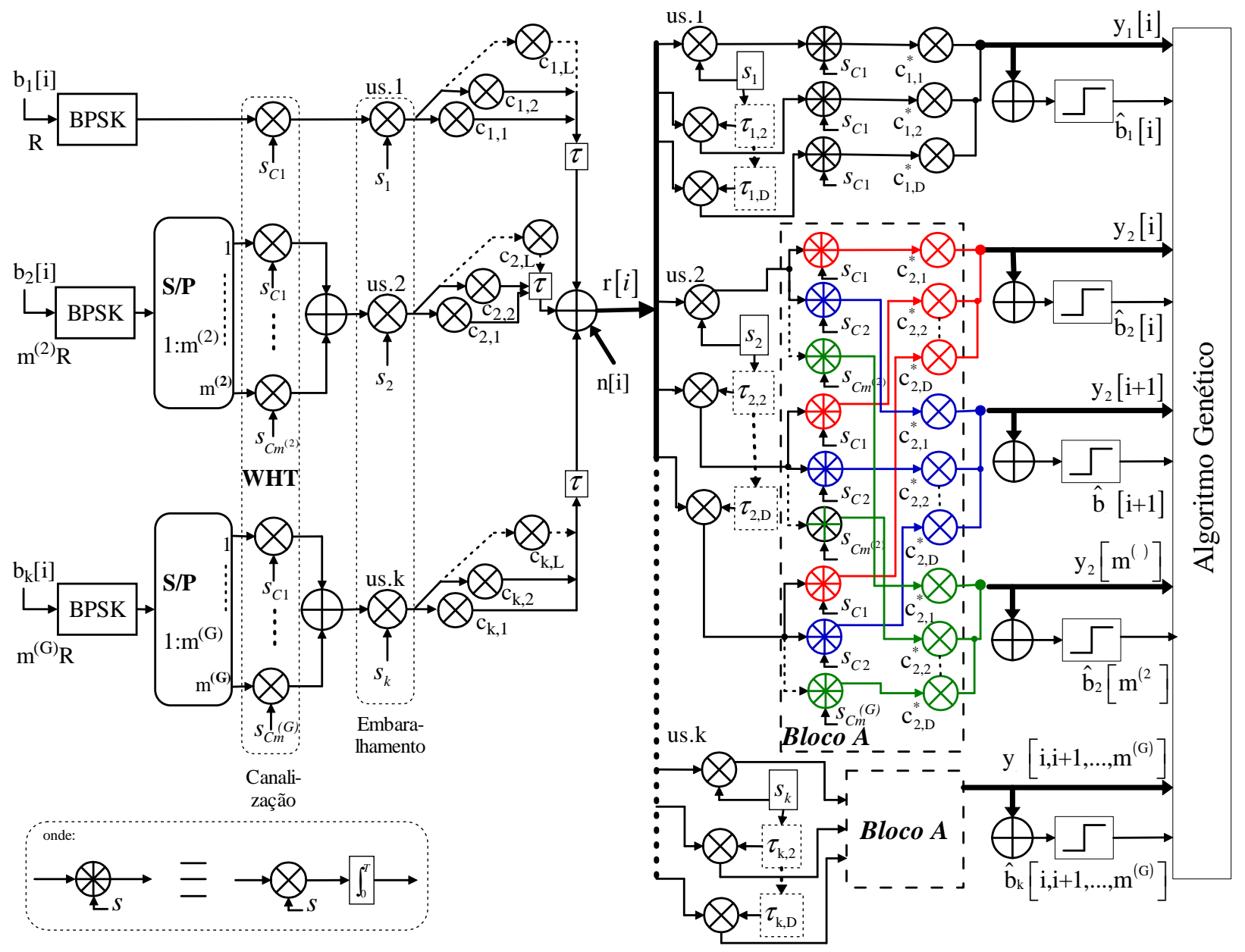

Figura 1: Sistema DS/CDMA multitaxa MC com detecção GA-MuD

percursos independentes com desvanecimento Rayleigh, o sinal em banda base recebido (assumindo filtro passa-baixa ideal) na estação rádio-base é:

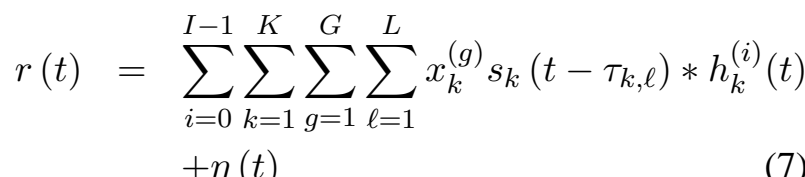

onde $K$ é o número de usuários físicos multitaxa ativos no sistema, $t \in[0, T]$, a amplitude $A_{k}$ é assumida constante ao longo dos $I$ bits transmitidos, $b_{k} \in\{ \pm 1\}$ é o bit de informação transmitido, $s_{k}$ é uma réplica da sequiência de assinatura PN atribuída ao $k$-ésimo usuário sendo $\tau_{k, \ell} \mathrm{o}$ atraso aleatório correspondente; este atraso considera a natureza assíncrona da transmissão, $d_{k}$, como também o atraso de propagação, $\Delta_{k, \ell}$ para o $k$-ésimo usuário, $\ell$-ésimo percurso, resultando em $\tau_{k, \ell}=\Delta_{k, \ell}+d_{\ell}$; $\eta(t)$ representa o AWGN (Additive White Gaussian Noise) com densidade de potência bilateral igual a $N_{0} / 2$ e a resposta impulsiva do canal para o $k$-ésimo usuário MC no intervalo do $i$-ésimo bit pode ser escrita como:

$$
h_{k}^{(i)}(t)=\sum_{\ell=1}^{L} c_{k, \ell}^{(i)} \delta\left(t-\Delta_{k, \ell}\right)
$$

onde $c_{k, \ell}^{(i)}=\beta_{k, \ell}^{(i)} e^{j \phi_{k, \ell}^{(i)}}$ indica o coeficiente complexo do canal para o $k$-ésimo usuário $\mathrm{MC}, \ell$ ésimo percurso; assume-se que a fase de $c_{k}$ terá uma distribuição uniforme em $\phi_{k, \ell} \in[0,2 \pi)$ 
e o módulo do canal $\beta_{k, \ell}$ representa o desvanecimento de pequena escala com envoltória seguindo uma distribuição Rayleigh. Adicionalmente, considerou-se ganho de canal normalizado para todos os usuários, ou seja, $E\left[\sum_{\ell=1}^{L}\left|c_{k, \ell}^{(i)}\right|^{2}\right]=1$ para $k=1,2, \ldots, K$. Utilizando notação vetorial, a equação (7) pode ser re-escrita:

$$
r(t)=\sum_{i=0}^{I-1} \mathbf{s}^{T}(t-i T) \mathbf{a c}^{(i)} \mathbf{u}^{(i)}+\eta(t)
$$

onde: $\quad \mathbf{s}(t)=\left[s_{1}\left(t-\tau_{1,1}\right), s_{1}\left(t-\tau_{1,2}\right), \ldots\right.$, $\left.s_{1}\left(t-\tau_{1, L}\right), \ldots, s_{k}\left(t-\tau_{k, \ell}\right), \ldots, s_{K}\left(t-\tau_{K, L}\right)\right]^{T}$ é a matriz de seqüência de espalhamento PN de todos os usuários $\mathrm{MC}$, $\mathbf{a}=\operatorname{diag}\left[\sqrt{P_{1}^{\prime}} \mathbf{I}, \sqrt{P_{2}^{\prime}} \mathbf{I}, \ldots, \sqrt{P_{K}^{\prime}} \mathbf{I}\right]$ é a matriz diagonal das amplitudes recebidas dos usuários, incluindo os efeitos de perdas de percurso e sombreamento do canal, onde $\mathbf{I}_{L \times L}$ é a matriz identidade de dimensão $L ; \mathbf{c}^{(i)}=\operatorname{diag}\left[c_{1,1}^{(i)}, \ldots, c_{1, L}^{(i)}, c_{2,1}^{(i)}\right.$, $\left.\ldots, c_{2, L}^{(i)}, \ldots, c_{K, L}^{(i)}\right]$ é a matriz diagonal de ganho de canal, e o vetor de bits espalhado pelo códigos de canalização MC é dado por $\mathbf{u}^{(i)}=\left[\mathbf{u}_{1}^{(i)}, \mathbf{u}_{2}^{(i)}, \ldots, \mathbf{u}_{K}^{(i)}\right]^{T}$ com $\mathbf{u}_{k}^{(i)}$ o vetor contendo réplicas (multipercurso) do $i$-ésimo bitcanalizado (dado por (2)) do $k$-ésimo usuário físico multitaxa MC de dimensão $1 \times L$. Por simplicidade e sem perda de generalidade, considerou-se atrasos aleatórios ordenados, ou seja, $0=\tau_{1,1} \leq \tau_{1,2} \leq$ $\cdots \tau_{1, L} \leq \tau_{2,1} \leq \cdots \leq \tau_{K, L}<T$.

Para canais com desvanecimento multipercurso e esquema multitaxa MC, o receptor Rake consiste de um banco de K.D filtros casados às sequências de embaralhamento dos usuários físicos MC, com ordem de diversidade de percurso $D \leq L$, seguido do segundo desespalhamento (canalização) objetivando recuperar os $m^{(g)}$ bits transmitidos simultaneamente nos canais paralelos. Assim, as $m^{(g)}$ saídas do filtro casado para o $k$-ésimo usuário físico MC correspon- dente ao $\ell$-ésimo componente multipercurso (ramo), amostrado ao final do período básico $T$ do $i$-ésimo intervalo de bit pode ser expresso como: $y_{k, \ell}^{(i)}[m]=$

$$
\begin{aligned}
& =\frac{1}{\sqrt{N_{C}}} \int_{0}^{T} r(t) s_{k}\left(t-i T-\tau_{k, \ell}\right) s_{C k, m}^{(g)}(t) d t \\
& =\sqrt{P_{k}^{\prime}} T \beta_{k, \ell}^{(i)} b_{k, m}^{(i)}+S I_{k, m, \ell}^{(i)}+I_{k, m, \ell}^{(i)}+n_{k, \ell}^{(i)}
\end{aligned}
$$

onde $m=1,2, \ldots, m^{(g)}$; o primeiro termo corresponde ao sinal desejado, o segundo termo à auto-interferência (SI), o terceiro à interferência de múltiplo acesso (MAI) sobre o $\ell$-ésimo componente multipercurso do $m$-ésimo canal paralelo do $k$ ésimo usuário, e o último o AWGN filtrado. Os termos SI e MAI dependem da função de correlação parcial, que no caso do duplo espalhamento do esquema $\mathrm{MC}$ resulta:

$\mathcal{R}_{u, m, k, n}(\tau, i)=\int_{0}^{T} s_{C u, m}^{(g)} s_{u}(t) s_{C k, n}^{(g)} s_{k}(t+i T+\tau) d t$

com os índices $m$ e $n$ indicando os respectivos canais paralelos do $u$-ésimo e $k$-ésimo usuário físico MC.

Utilizando notação vetorial, a saída do banco de filtros casados para o $i$-ésimo intervalo de bit, considerando $D$ ramos de diversidade de percurso, é dado por pela equação (11):

As matrizes $\mathbf{R}[0]$ e $\mathbf{R}[1]$, de dimensão $D \mathcal{K}_{v} \times$ $D \mathcal{K}_{v}$, são definidas pelos elementos:

$R_{i j}[0]=\left\{\begin{array}{cl}1 & , \text { se } u=k \text { e } m=n \\ \mathcal{R}_{u, m, k, n}\left(\tau_{u k}, 0\right) & , \quad \text { se } u<k \\ \mathcal{R}_{k, n, u, m}\left(\tau_{u k}, 0\right) & , \text { se } u>k \\ \mathcal{R}_{u, m, k, n}\left(\tau_{u k}, 0\right) & , \quad \text { se } u=k \text { e } m<n \\ \mathcal{R}_{k, n, u, m}\left(\tau_{u k}, 0\right) & , \quad \text { se } u=k \text { e } m>n\end{array}\right.$

$$
R_{i j}[1]=\left\{\begin{array}{cc}
0 & , \quad \text { se } u \geq k \\
\mathcal{R}_{k, n, u, m}\left(\tau_{u k}, 0\right) & , \quad \text { se } u<k
\end{array}\right.
$$

onde $i=u . m$ e $j=k . n, \mathcal{K}_{v}=\sum_{g=1}^{G} K^{(g)} m^{(g)}$ é o número de usuários virtuais do sistema MC 


$$
\begin{aligned}
\mathbf{y}^{(i)} & =\left[y_{k, 1,1}^{(i)}, y_{k, 2,1}^{(i)}, \ldots, y_{k, D, 1}^{(i)}, \ldots, y_{k, 1,2}^{(i)}, \ldots, y_{k, D, 2}^{(i)} \ldots, y_{k, 1, m^{(G)}}^{(i)}, y_{k, 2, m^{(G)}}^{(i)}, \ldots, y_{k, D, m^{(G)}}^{(i)}\right] \\
& =\mathbf{R}^{T}[1] \underline{\mathbf{a c}}^{(i+1)} \mathbf{b}^{(i+1)}+\mathbf{R}[0] \underline{\mathbf{a c}}^{(i)} \mathbf{b}^{(i)}+\mathbf{R}[1] \underline{\mathbf{a c}}^{(i-1)} \mathbf{b}^{(i-1)}+\mathbf{n}^{(i)}
\end{aligned}
$$

onde:

$$
\begin{gathered}
\underline{\mathbf{b}}^{(i)}=\left[b_{k, 1,1}^{(i)}, b_{k, 2,1}^{(i)}, \ldots, b_{k, D, 1}^{(i)}, \ldots, b_{k, 1,2}^{(i)} \ldots, b_{k, D, 2}^{(i)} \ldots, b_{k, 1, m^{(G)}}^{(i)}, b_{k, 2, m^{(G)}}^{(i)}, \ldots, b_{k, D, m^{(G)}}^{(i)}\right]^{T} \\
\underline{\mathbf{c}}^{(i)}=\operatorname{diag}\left[\mathrm{c}_{\mathrm{k}, 1,1}^{(\mathrm{i})}, \mathrm{c}_{\mathrm{k}, 2,1}^{(\mathrm{i})}, \ldots, \mathrm{c}_{\mathrm{k}, \mathrm{D}, 1}^{(\mathrm{i})}, \ldots, \mathrm{c}_{\mathrm{k}, 1,2}^{(\mathrm{i})}, \ldots, \mathrm{c}_{\mathrm{k}, \mathrm{D}, 2}^{(\mathrm{i})} \ldots, \mathrm{c}_{\mathrm{k}, 1, \mathrm{~m}}^{(\mathrm{i})}, \mathrm{c}_{\mathrm{k}, 2, \mathrm{~m}(\mathrm{G})}^{(\mathrm{i})}, \ldots, \mathrm{c}_{\mathrm{k}, \mathrm{D}, \mathrm{m}(\mathrm{G})}^{(\mathrm{i})}\right] \\
\underline{\mathbf{a}}^{(i)}=\operatorname{diag}\left[\mathrm{a}_{\mathrm{k}, 1}^{(\mathrm{i})} \mathbf{I}, \ldots, \mathrm{a}_{\mathrm{k}, 2}^{(\mathrm{i})} \mathbf{I}, \ldots, \mathrm{a}_{\mathrm{k}, \mathrm{m}^{(\mathrm{G})}}^{(\mathrm{i})} \mathbf{I}\right]
\end{gathered}
$$

com $k \in K^{(g)}$ e $\mathbf{I}_{D \times D}$ é a matriz identidade de dimensão $D$.

DS/CDMA. O Rake consiste em combinar as saídas disponíveis do banco de filtros casados para cada usuário (fingers) de forma coerente e ponderada pelos respectivos ganhos de canal (PROAKIS, 1995). O combinador de razão máxima (MRC - Maximal Ratio Combiner) combina os sinais dos $D$ correlatores:

$$
z_{k}^{(i)}=\sum_{\ell=1}^{D} \operatorname{Re}\left\{y_{k, \ell}^{(i)} \hat{\beta}_{k, \ell}^{(i)}\right\}
$$

seguido de um circuito de decisão abrupta, $\hat{b}_{k}^{(i)}=$ $\operatorname{sign}\left(z_{k}^{(i)}\right)$.

O desempenho obtido com o receptor Rake será consideravelmente deteriorado quando o número de usuários compartilhando o mesmo canal crescer (aumentando a MAI) e/ou quando a potência dos usuários interferentes aumentar (near-far ef$f e c t)$. O melhor desempenho, dentre os detectores $\mathrm{MuD}$, é alcançado com o detector $\mathrm{OMuD}$ (VERDÚ, 1998), sendo baseado na estratégia de máxima verossimilhança. Neste contexto, o vetor de máxima verossimilhança que deve ser encontrado pelo $\mathrm{OMuD}$ possui dimensão $\mathcal{K}_{v} D I$, sendo escrito por:

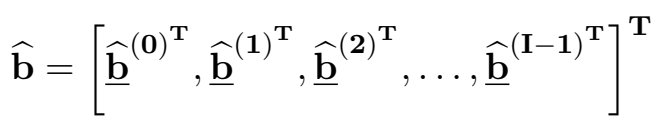

Baseado na observação do MFB, vetor $\mathbf{y}^{(i)}$ em (11), encontrar o vetor de máxima verossimilhança $\widehat{b}$ é equivalente a selecionar o vetor de bits $\mathcal{B}$ que maximiza a chamada função de verossimilhança (LLF) (VERDÚ, 1998):

$$
\Omega(\mathcal{B})=2 \operatorname{Re}\left\{\mathcal{B}^{T} \mathcal{C}^{H} \mathcal{A} \mathcal{Y}\right\}-\mathcal{B}^{T} \mathcal{C} \mathcal{A} \mathbf{R} \mathcal{A C}^{H} \mathcal{B}
$$

onde as matrizes diagonais coeficientes de canal e amplitudes, de dimensão $\mathcal{K}_{v} I D$, são definidas, respectivamente, por:

$$
\begin{gathered}
\mathcal{C}=\operatorname{diag}\left[\underline{\mathbf{c}}^{(0)}, \underline{\mathbf{c}}^{(1)}, \underline{\mathbf{c}}^{(2)}, \ldots, \underline{\mathbf{c}}^{(\mathrm{I}-1)}\right] \quad \mathrm{e} \\
\mathcal{A}=\operatorname{diag}\left[\underline{\mathbf{a}}^{(\mathbf{0})}, \underline{\mathbf{a}}^{(\mathbf{1})}, \underline{\mathbf{a}}^{(\mathbf{2})}, \ldots, \underline{\mathbf{a}}^{(\mathbf{I}-\mathbf{1})}\right],
\end{gathered}
$$

$\mathcal{Y}=\left[\mathbf{y}^{(0)}, \mathbf{y}^{(1)}, \mathbf{y}^{(2)}, \ldots, \mathbf{y}^{(I-1)}\right]^{T}$ é a matriz saída do banco de filtros casados, composta por $I$ vetores $\mathbf{y}^{(i)}$ em (11); o operador Hermitiano transposto é definido por $(\cdot)^{H}=\left[(\cdot)^{*}\right]^{T}$ e a blocktridiagonal, block-Toeplitz matriz de correlação $\mathbf{R}$, com dimensão de $\mathcal{K}_{v} I D \times \mathcal{K}_{v} I D$ é definida como 
(VERDÚ, 1998):

$$
\mathbf{R}=\left[\begin{array}{cccccc}
\mathbf{R}[0] & \mathbf{R}^{T}[1] & \mathbf{0} & \cdots & \mathbf{0} & \mathbf{0} \\
\mathbf{R}[1] & \mathbf{R}[0] & \mathbf{R}^{T}[1] & \cdots & \mathbf{0} & \mathbf{0} \\
\mathbf{0} & \mathbf{R}[1] & \mathbf{R}[0] & \cdots & \mathbf{0} & \mathbf{0} \\
\cdots & \cdots & \cdots & \ddots & \cdots & \cdots \\
\mathbf{0} & \mathbf{0} & \mathbf{0} & \cdots & \mathbf{R}[1] & \mathbf{R}[0]
\end{array}\right]
$$

Portanto, o quadro completo de bits transmitidos estimados para todos os $K$ usuários físicos pode ser obtido otimizando (19), resultando em:

$$
\widehat{\mathbf{b}}=\arg \left\{\max _{\mathcal{B} \in\{ \pm 1\}^{\mathcal{K}_{v} I}}[\Omega(\mathcal{B})]\right\}
$$

O OMuD consiste na busca do melhor vetor de bits de dados em um conjunto com todas as possibilidades, ou seja, é um problema com combinação NP-completa (VERDÚ, 1989), no qual os algoritmos tradicionais são ineficientes. Portanto, a utilização do GA-MC-MuD para esse tipo de problema mostrase atraente, pois é possível obter soluções ótimas (ou próximas) utilizando espaços de busca reduzidos. Desta forma, O GA-MC-MuD procurará maximizar a LLF testando distintos vetores de bits candidatos a cada nova geração; tais tentativas buscam maximizar o desempenho médio do sistema MC DS/CDMA, aproximando ou mesmo igualando o obtido pelo detector OMuD.

\section{Algoritmo Genético}

Este algoritmo procura descrever as interações biológicas existentes entre as células de um organismo, alterando o genótipo na etapa de transferência do material genético. Neste caso, por se tratar de reprodução de células, os operadores genéticos mutação e crossover são utilizados como estratégias de diversificação (GOLDBERG, 1989; MITCHELL, 1998). Uma descrição genérica apresentando as diferentes etapas do algoritmo genético aplicado a detecção multiusuário multitaxa (GA-MC-MuD) é mostrado a seguir.

Entradas: $p, \mathcal{B}_{1}, M, G_{T} \quad$ Saídas: $\mathcal{B}_{1}$

1. Inicializa primeira população $\mathcal{B} ; x=0$;

2. Calcula a $\operatorname{LLF}(\mathcal{B})$;

3. Enquanto $x<G_{T}$ faça;

4. $\quad \mathcal{B}_{\text {selected }}=\operatorname{Seleção}(\mathcal{B}, M)$;

5. $\mathcal{B}_{\text {cross }}=\operatorname{Crossover}\left(\mathcal{B}_{\text {selected }}\right)$;

6. $\quad \mathcal{B}_{\text {new }}=\operatorname{Mutação}\left(\mathcal{B}_{\text {cross }}\right)$;

7. Calcula a $\operatorname{LLF}\left(\mathcal{B}_{\text {new }}\right)$;

8. $\mathcal{B}=\operatorname{Reposição~}\left(\mathcal{B} \cup \mathcal{B}_{\text {new }}\right)$;

9. $x=x+1$;

10. fim

Para uma análise sistemática considerando inúmeras abordagens heurísticas no processo de detecção multiusuário em sistemas DS/CDMA de taxa única, canais AWGN e Rayleigh plano, veja (CIRIACO; ABRÃO; JESZENSKY, 2005).

O universo total de busca do GA-MC-MuD é caracterizado por todas as possíveis combinações de dados recebidos que apresentam um mesmo bit para todos os $D$ ramos de processamento, ou seja, $\widehat{b}_{k, 1, m^{(g)}}^{(i)}=\widehat{b}_{k, 2, m^{(g)}}^{(i)}=\ldots=\widehat{b}_{k, D, m^{(g)}}^{(i)} \in\{ \pm 1\}$. Isso significa que o universo de busca do algoritmo genético para o problema da detecção multiusuário é independente do número de percursos. Para soluções via algoritmos genéticos, a escolha do tamanho da população $(p)$ é um fator importante para o custo computacional e a qualidade das soluções obtidas. Este trabalho utiliza uma equação adaptada de (AHN; RAMAKRISHNA, 2002) para encontrar o tamanho da população $p$ adequada ao problema da detecção multiusuário:

$$
p=10 \cdot\left\lfloor 0,3454\left(\sqrt{\pi\left(\mathcal{K}_{v} \cdot I-1\right)}+2\right)\right\rfloor
$$

onde o operador $\lfloor$.$\rfloor retorna o menor inteiro. Esta$ equação é calculada no estágio de inicialização do 
GA-MC-MuD e mantida constante para todas as gerações. As estimativas do receptor Rake são utilizadas como o indivíduo inicial. Os outros membros da primeira população $\mathcal{B}_{1}$ são obtidos a partir do indivíduo inicial com perturbações convenientes (YEN; HANZO, 2004; ABRÃO; CIRIACO; JESZENSKY, 2004). No contexto MuD a aptidão é medida através da função LLF (19) sendo diretamente responsável pela morte ou vida dos indivíduos. O tamanho do chamado 'mating pool' $(M)$ deve ser escolhido visando garantir a velocidade de convergência e a qualidade da solução final (GOLDBERG, 1989). Para o problema $\mathrm{MuD}$ adotou-se $M=0.1 p$. Neste trabalho, o processo de seleção seleciona os melhores $M$ indivíduos da população de tamanho $p$ para serem os pais da próxima geração. Conseqüentemente, os $p-M$ indivíduos com menores valores para a função custo são removidos do estágio de reprodução.

Os operadores genéticos são necessários para que a população se diversifique e mantenha características de adaptação adquiridas nas gerações anteriores. Estes transformam a população através de sucessivas gerações, estendendo a busca até chegar a um resultado satisfatório.

O algoritmo GA utiliza o operador crossover como seu principal operador genético no intuito de trazer variabilidade de busca sem perder as características de adaptação adquiridas. A mutação não é considerada essencial, pois em uma população real a taxa de mutação é baixa, fazendo com que a mutação seja um mecanismo secundário na adaptação dos algoritmos genéticos. Para os operadores genéticos (MITCHELL, 1998), adotou-se crossover do tipo uniforme com probabilidade de crossover $p_{c}$ e de mutação $p_{m}$ baseada no ruído:

$$
\text { novo }_{\text {indiv }}=\operatorname{sign}\left(\operatorname{indiv}+\mathcal{N}\left(0, \sigma^{2}\right)\right)
$$

onde $\operatorname{sign}($.$) representa a função sinal e \mathcal{N}\left(0, \sigma^{2}\right)$ representa uma distribuição Gaussiana com média zero e desvio padrão $\sigma$. O valor do desvio padrão está fortemente relacionado com a taxa de mutação média (ABRÃO; CIRIACO; JESZENSKY, 2004). Este trabalho utiliza uma estratégia de reposição chamada 'elitismo do tipo global', onde apenas os melhores $p$ indivíduos da população conjunta de pais e filhos são mantidos para a próxima geração. Finalmente, o processo de otimização para o GA-MC-MuD é encerrado após um número fixo de gerações $\left(G_{T}\right)$.

\section{Resultados Numéricos}

Foram realizadas simulações computacionais Monte-Carlo visando comprovar a eficiência do algoritmo proposto. Em todas as simulações adotou-se os seguintes parâmetros: sequências de canalização do tipo Walsh-Hadamard de comprimento $N_{C}=8$; sequências de embaralhamento do tipo aleatória (PN) com ganho de processamento $N_{P N}=T / T_{c}=400$; canal Rayleigh lento com três percursos sendo o segundo e terceiro raios atrasados de $12 T_{c}$ e $24 T_{c}$ do primeiro raio respectivamente. Perfil atraso-potência exponencial com $E\left[\beta_{1}^{2}\right]=0,8047, E\left[\beta_{2}^{2}\right]=0,1625$ e $E\left[\beta_{3}^{2}\right]=0,0328$. Este perfil atraso-potência com um reduzido número de percursos, inspirado no estudo de canal COST 207 e sintetizado em (STUBER, 2001), foi adotado visando aliviar o tempo e complexidade computacional, viabilizando a execução das simulações computacionais Monte Carlo.

No intuito de simular a mobilidade dos usuários, considerou-se que os $K$ usuários físicos se movimentam com uma velocidade uniformemente distribuída no intervalo de $\left[0 ; v_{\max }\right]$, onde $v_{\max }=$ $100 \mathrm{~km} / \mathrm{h}$, resultando em uma frequência Doppler máxima de $f_{m}=\frac{v_{\max }}{\lambda_{c}}=185,18 \mathrm{~Hz}$ para uma frequência de portadora de $f_{c}=\frac{1}{\lambda_{c}}=2 \mathrm{GHz}$; adotou-se banda do sistema $B W=3,84 M h z$ e di- 
versidade Rake igual ao número de percursos, $D=$ $L=3$.

Para simular os diferentes tipos de serviços oferecidos, considerou-se dois sistemas onde os usuários transmitem com taxas de $8 R, 4 R, 2 R$ e $R$, com a taxa básica $R=9,6 \mathrm{kbps}$. Estes sistemas são descritos na tabela 1. Assim, para os dois sistemas simulados, o número total de usuários virtuais foi fixado $\mathrm{em} \mathcal{K}_{t}=m^{(1)} \cdot K^{(1)}+m^{(2)} \cdot K^{(2)}+m^{(3)} \cdot K^{(3)}=24$.

Tabela 1: Parâmetros dos sistemas multitaxa.

\begin{tabular}{|c|c|c|c|c|}
\hline Serv. & Us. Físico & Taxa & Us. Físico & Taxa \\
\hline S1 & $K^{(1)}=8$ & $R$ & $K^{(1)}=8$ & $R$ \\
\hline $\mathbf{S 2}$ & $K^{(2)}=2$ & $4 R$ & $K^{(2)}=4$ & $2 R$ \\
\hline S3 & $K^{(3)}=1$ & $8 R$ & $K^{(3)}=2$ & $4 R$ \\
\hline & \multicolumn{2}{|c|}{ Sistema 1} & \multicolumn{2}{|c|}{ Sistema 2} \\
\hline
\end{tabular}

Para a geração dos coeficientes de canal adotouse o modelo de Gans modificado (SILVA; ABRÃO; JESZENSKY, 2004), com coeficientes gerados no domínio da frequência. A relação $E_{b} / N_{0}$ média na entrada do receptor é dada por:

$$
\bar{\gamma}=\sum_{\ell=1}^{L} \bar{\gamma}_{\ell}, \quad \text { onde } \quad \bar{\gamma}_{\ell}=\frac{E_{b}}{N_{0}} E\left[\beta_{\ell}^{2}\right]
$$

Para efeito de comparação, o desempenho do detector Rake e o limite quando há apenas um único usuário com taxa $R$ ativo no sistema (SuB) (PROAKIS, 1995) foram incluídos. Os valores adotados para os parâmetros do GA-MC-MuD estão sintetizados na tabela 2, considerando os dois sistemas simulados. Em todas as simulações, o GA-MC-MuD processa e otimiza um conjunto de $\mathcal{K}_{v} . I . D$ bits por vez, sendo $I=5$ bits/usuário virtual e $G_{T}=40$. Os parâmetros potência de transmissão, ganho de canal (módulo e fase) e atraso dos usuários são perfeitamente conhecidos no receptor; adicionalmente, admite-se um sistema com controle perfeito de potência, ou seja, $P_{1}^{\prime}=P_{2}^{\prime}=\ldots=P_{K}^{\prime}$. Em todas as simulações Monte-Carlo adotou-se um número mínimo de erros por ponto de desempenho igual a 15 .
Tabela 2: Parâmetros do GA-MC-MuD.

\begin{tabular}{cccc|cccc}
\hline$p$ & $p_{m}$ & $p_{c}$ & $M$ & $p$ & $p_{m}$ & $p_{c}$ & $M$ \\
\hline 140 & $1,3 \%$ & $50 \%$ & 14 & 140 & $1,02 \%$ & $50 \%$ & 14 \\
\hline \multicolumn{3}{c}{ Sistema 1 } & \multicolumn{5}{c}{ Sistema 2} \\
\hline
\end{tabular}

A figura 2 mostra o desempenho encontrado pelo GA-MC-MuD na condição do sistema 1. Percebese que a BER para o algoritmo GA-MC-MuD aproxima-se do desempenho $\mathrm{SuB}$, evidenciando um enorme ganho de desempenho em relação ao detector Rake para todos os 3 grupos multitaxa avaliados.

A figura 3 mostra a curva de convergência do algoritmo GA-MC-MuD nas condições do sistema 1; este gráfico expressa a evolução de desempenho do algoritmo heurístico GA-MC-MuD em direção à solução ótima (obtida via $\mathrm{OMuD}$ ), em função do aumento do número de gerações computadas. Evidencia-se a rápida convergência para o limite $\mathrm{SuB}$ dos 3 grupos multitaxa. Percebe-se que com pouquíssima gerações $\left(G_{T} \approx 25\right)$ o algoritmo GAMC-MuD já é capaz de encontrar a solução quaseótima, implicando em uma enorme redução da complexidade quando comparada a do OMuD. Em valores numéricos, esta redução de complexidade pode ser obtida verificando o número de soluções candidatas testadas (universo de busca visitado) por ambos os detectores: ao final de $G_{T}=40$ gerações, o algoritmo GA-MC-MuD testou p. $G_{T}=5600$ soluções candidatas, enquanto que para o OMuD seriam necessários $2^{\mathcal{K}_{v} . I}=1,4.10^{36}$ testes. Estes testes não foram computados, devido à impossibilidade de realização utilizando computadores pessoais atuais de alto desempenho e capacidade de armazenamento. figura 4 mostra o desempenho do GA-MCMuD na condição do sistema 2. Neste caso, existem 2 usuários para o grupo de maior taxa de dados; mesmo nesta condição de maior interferência, o desempenho médio após $G_{T}=40$ gerações é bastante próximo ao limite $\mathrm{SuB}$ para todos os 3 serviços avaliados. Esta figura corrobora a idéia de eficiência do 


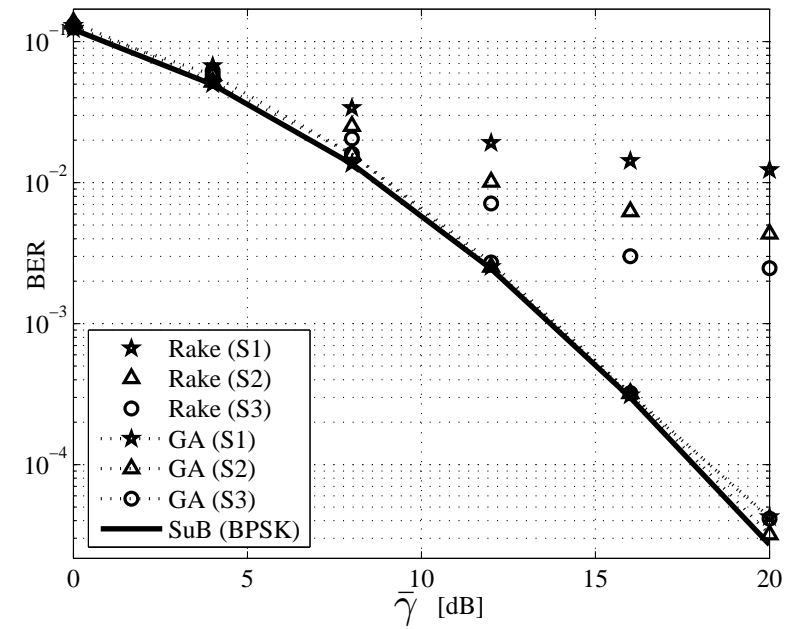

Figura 2: BER para os detectores Rake e GA-MCMuD com diferentes taxas de transmissão dadas pelo Sistema 1.

algoritmo GA-MC-MuD proposto aplicado aos sistemas MC DS/CDMA em canais com desvanecimento multipercurso, verificando-se uma ótima qualidade da solução final após $G_{T}=40$ gerações.

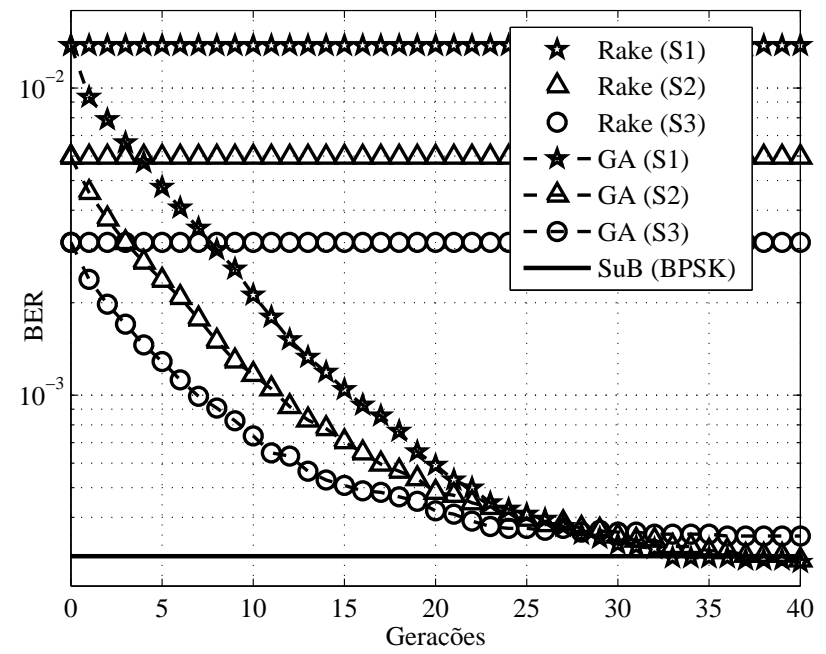

Figura 3: Convergência para o GA-MC-MuD; sis-

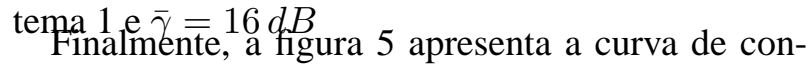
vergência do algoritmo GA-MC-MuD, na condição do sistema 2, em função do número de gerações computadas. Aumentando-se o número de usuários do grupo de maior taxa e incrementado-se em $2 d B$ a

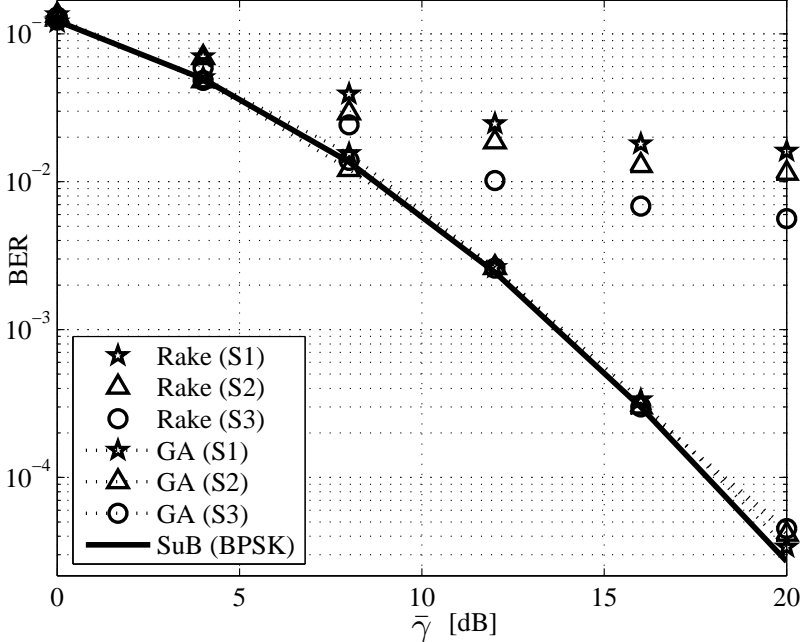

Figura 4: BER para os detectores Rake e GA-MCMuD com diferentes taxas de transmissão dadas pelo Sistema 2.

potência recebida de todos os usuários (em relação às condições de operação da figura 3), percebe-se que ainda com poucas gerações $\left(G_{T}=40\right)$ o algoritmo GA-MC-MuD é capaz de atingir a solução ótima ou quase-ótima, implicando em uma redução da complexidade quando comparada à do OMuD.

\section{Complexidade Computacional}

No intuito de se encontrar a redução da complexidade do algoritmo GA-MC-MuD em relação ao $\mathrm{OMuD}$ de forma mais precisa, é necessário que a complexidade do GA-MC-MuD e do OMuD seja expressa em termos do número de operações relevantes em termos de flops (float point operations) (Higham, 1996). Para isso, deve-se encontrar o número de operações realizadas a cada cálculo da função custo (19). Para o cálculo da função custo, equação (19), o conjunto de operações $\mathcal{F}_{1}=\mathcal{C}^{H} \mathcal{A} \mathcal{Y}$ e $\mathcal{F}_{2}=\mathcal{C} \mathcal{A} \mathbf{R} \mathcal{A C}^{H}$ podem ser obtidas antes do laço de otimização de cada algoritmo. Para cada teste da solução candidata, as operações $\mathcal{F}_{1} \mathcal{B}$ e $\mathcal{B}^{T} \mathcal{F}_{2} \mathcal{B}$ são computadas, o que em termos de operações é equi- 


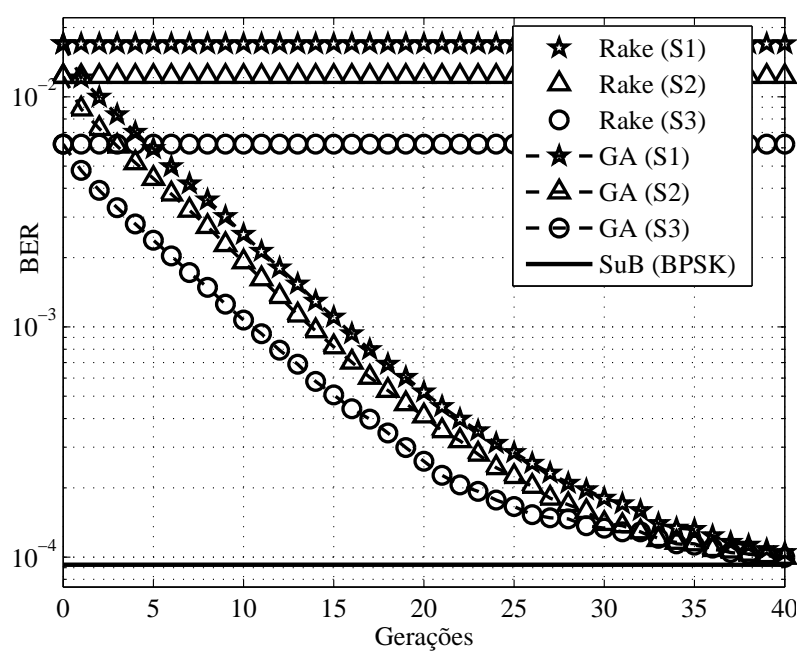

Figura 5: Convergência para o GA-MC-MuD; sistema 2 e $\bar{\gamma}=18 d B$.

valente a $\left(\mathcal{K}_{v} \cdot I . D\right)^{2}+2 \mathcal{K}_{v} \cdot I . D$ multiplicações e 1 transposição de ordem $\mathcal{K}_{v}$.I.D. Para o $\mathrm{OMuD}$, o número de operações cresce exponencialmente com o número de usuários, i.e., $\mathcal{O}\left(2^{\mathcal{K}_{v} . I}\left(\mathcal{K}_{v} . I . D\right)^{2}\right)$. De forma mais precisa, $2^{\mathcal{K}_{v} \cdot I}$ gerações de vetores de bits de ordem $\mathcal{K}_{v} . I . D$ e $2^{\mathcal{K}_{v} . I}$ cálculos da função custo são necessárias para a detecção simultânea de um conjunto de $I$ bits dos $\mathcal{K}_{v}$ usuários virtuais. Para o GA-MuD o número de operações cresce dependendo da relação $\mathcal{O}\left(p . G_{T}\left(\mathcal{K}_{v} \cdot I . D\right)^{2}\right)$, sendo computadas $3 p . G_{T}+p-1$ gerações de vetores de bits de ordem $\mathcal{K}_{v} . I . D, M . G_{T}$ seleções de vetores de bits de ordem $\mathcal{K}_{v} . I . D, p . G_{T}+p$ cálculos da função custo, $3 p . G_{T}$ ordenações de vetores de ordem $\mathcal{K}_{v} . I . D, p . G_{T}$ comparações de vetores de ordem $\mathcal{K}_{v} . I . D$ e $p . G_{T}$ troca de vetores de bit de or$\operatorname{dem} \mathcal{K}_{v}$.I.D. Como os valores numéricos para as variáveis $\mathcal{K}_{v}, I, D, p, G_{T}$ e $M$ dos sistemas 1 e 2 são idênticos, obtêm-se um mesmo valor para a complexidade computacional dos detectores $\mathrm{OMuD}$ e GAMC-MuD nas duas condições de sistema simulados. Neste caso, o detector OMuD necessita computar aproximadamente $1,72.10^{41}$ operações para detectar de forma ótima um conjunto de $I$ bits dos $\mathcal{K}_{v}$ usuários virtuais. Em contraste ao $\mathrm{OMuD}$, o GAMC-MuD necessita computar apenas $\approx 7,26.10^{8}$ operações, resultando em redução na complexidade computacional.

\section{Conclusões}

Este trabalho analisou a técnica heurística de otimização combinatória baseada em algoritmos genéticos aplicada ao problema da detecção multiusuário quase-ótima em sistemas de múltiplo acesso DS/CDMA multitaxa MC, em canais Rayleigh lento multipercurso visando oferecer uma diversidade de serviços multimídia e ao mesmo tempo garantir a qualidade desses serviços. $\mathrm{O}$ algoritmo GA-MC-MuD apresentou desempenho muito próximo ao limite SuB para as duas condições de sistema analisadas, i.e, usuários físicos distribuídos em três taxas de transmissão, resultando em 24 usuários virtuais.

O algoritmo heurístico GA-MC-MuD oferece a vantagem de uma drástica redução de complexidade computacional em relação ao $\mathrm{OMuD}$ com uma perda de desempenho apenas marginal, mesmo em canais agressivos; estas características resultam em um ótimo compromisso desempenho-complexidade, viabilizando a implementação do algoritmo GA-MC$\mathrm{MuD}$ na estação rádio-base de sistemas $3 \mathrm{G}$ e $4 \mathrm{G}$.

\section{Referências}

ABRÃO, T. Canceladores de Interferência Multiusuário Aplicados a Sistemas DS/CDMA de Múltipla Taxa. 364 p. Tese (Doutorado) - Escola Politécnica da Universidade de São Paulo - EPUSP, São Paulo - SP, 2001.

ABRÃO, T.; CIRIACO, F.; JESZENSKY, P. J. E. Evolutionary programming with cloning and adaptive cost funciton applied to multi-user ds-cdma systems. In: 2004 IEEE International Symposium on Spread Spectrum Techniques and Applications. Sydney: Australia, 2004. (Adaptive Multiuser Detection), p. 160-163. ISBN 0-7803-8408-3. 
AHN, C. W.; RAMAKRISHNA, R. S. A genetic algorithm for shortest path routing problem and the sizing of populations. IEEE Transactions on Evolutionary Computation, v. 6, n. 6, p. 566-578, December 2002.

CIRIACO, F.; ABRÃO, T.; JESZENSKY, P. J. E. Algoritmos heurísticos aplicados à detecção multiusuário ds/cdma. Revista Brasileira de Telecomunicações, v. 20, n. 2, p. 37-51, 2005.

GOLDBERG, D. E. Genetic Algorithms in Search Optimization and Machine Learning. Nova York: AddisonWesley, 1989.

HIGHAM, N. J. Accuracy and Stability of Numerical Algorithms. Philadelphia, PA: SIAM, 1996.

JOHANSSON, A. L. Sucessive Interference Cancellation in DS-CDMA Systems. Tese (Doutorado) - Chalmers University of Technology, Göoteborg - Sweden, 1998.

MITCHELL, M. An Introduction to Genetic Algorithms. Cambridge: MIT Press, 1998.

PROAKIS, J. G. Digital Communications. 2. ed. New York: McGraw-Hill, 1995. (Electrical Engineering. Communications and Signal Processing). ISBN 0-07-0509379.

SILVA, V.; ABRÃO, T.; JESZENSKY, P. J. E. Statistically correct simulation models for the generation of multiple uncorrelated rayleigh fading waveforms. In: 2004 IEEE International Symposium on Spread Spectrum Techniques and Applications. Sydney: Australia, 2004. p. 1326-1329.

STUBER, G. L. Principles of Mobile Communication. 2. ed. Massachusetts: Kluwer Academic Publishers, 2001. ISBN 0-7923-7998-5.

VERDÚ, S. Computational complexity of optimum multiuser detection. Algorithmica, v. 4, p. 303-312, 1989.

VERDÚ, S. Multiuser Detection. USA: Cambridge University Press, 1998. ISBN 0-521-59373-5.

YEN, K.; HANZO, L. Genetic-algorithm-assisted multiuser detection in asynchronous cdma communications. IEEE Transactions on Vehicular Technology, v. 53, n. 5, p. 1413-1422, September 2004. 